\title{
Biomechanical modeling of brain soft tissues for medical applications
}

\author{
Fanny Morin $^{\mathrm{a}, \mathrm{b}}$, Matthieu Chabanas ${ }^{\mathrm{a}, *}$, Hadrien Courtecuisse ${ }^{\mathrm{b}}$, Yohan Payan ${ }^{\mathrm{a}}$ \\ ${ }^{a}$ TIMC-IMAG, Univ. Grenoble Alpes, CNRS, F-3800o Grenoble, France \\ ${ }^{b}$ AVR-ICube, Univ. Strasbourg, CNRS, F-6rooo Strasbourg, France
}

\begin{abstract}
For more than 60 years, many works have focused on the determination of the brain biomechanical properties. While the highly non linear behavior of the organ as well as the very low soft tissues stiffness are stressed out, no consensus is universally accepted. Variations in the reported constitutive laws and parameters may be due to the diversity of the experimental protocols and to patient peculiarities. In addition to these rheological properties, boundary conditions are at least as important. Especially, a low sensitivity of the model to these properties is observed when loads are imposed through displacements. For this reason and/or due to the small displacements observed and execution time requirements, most finite element brain models are simulated based on linear elastic law. Nevertheless, models with various boundary conditions have been proposed in the literature, being carefully designed for specific medical applications.

A survey about brain soft tissues biomechanical modeling is presented in this paper. The main works are then presented before describing a new vessel-based brain-shift compensation model using intra-operative Doppler ultrasound imaging.
\end{abstract}

Keywords Brain • Finite element modeling • Biomechanical properties • Constitutive laws • Boundary conditions $\bullet$ Medical simulation.

\section{Introduction: Clinical Context}

Nowadays, a growing number of brain disorders are diagnosed. In the US, 1.5 million of traumatic brain injuries were reported in 2003 (Rutland-Brown et al. 2006). In addition, while 77,670 new cases of primary tumors of the brain and spinal cord are estimated for 2016 (Ostrom et al. 2015), "the number of individuals over age 50 with Parkinson disease is expected to more than double from 4.1 million in 2005 to 8.7 million in 2030" (Dorsey et al., 2007). Brain disorders are thus one of the major public health issues.

For medical purposes, many groups have focused their research on the modeling of this organ. Relying on soft tissues characterization studies, several biomechanical models have been proposed

${ }^{*}$ Corresponding author: 凶matthieu.chabanas@imag.fr 


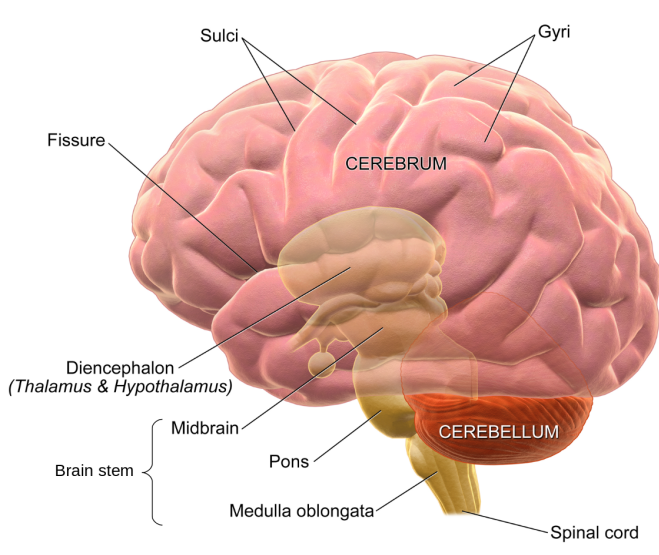

(a) Encephalon

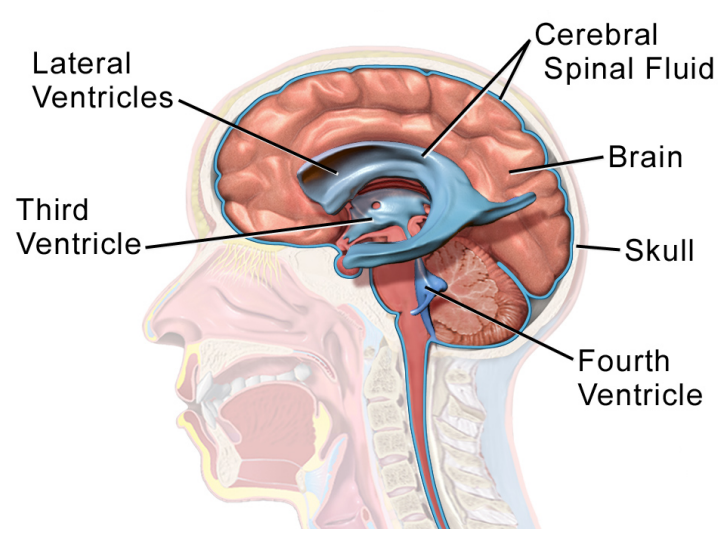

(b) Ventricular system

Figure 7.1: Anatomy of the brain (images adapted from "Blausen gallery 2014". Wikiversity Journal of Medicine. DOI:10.15347/wjm/2014.010. ISSN 20018762)

in the literature in order to predict or compensate for the intra-operative deformations of the brain, to visualize the effects of a pathological region growth or to train students to practice surgery. A review of these finite element $(\mathrm{FE})$ brain models with their biomechanical properties is proposed in this survey.

First, the brain anatomy is introduced in Section 2, Section 3 provides an overview of the brain models in the literature, detailing the characterization of mechanical properties, the importance of boundary conditions and then FE models in their applicative context. Finally, a model for craniotomy-induced brain-shift compensation, developed by the authors, is introduced in Sections 4 and 5 .

\section{Anatomical Description of the Brain}

This section gives an overview of the brain anatomy, especially the morphological structures of the organ. Their function is also mentioned for the understanding of the brain modeling concerns.

\subsection{Soft tissues}

The Central Nervous System (CNS) is composed of the encephalon, located within the skull, and of the spinal cord, situated in the spine. The encephalon is constituted of tree parts: the brainstem, the cerebellum and the cerebrum (see Figure 7.1 (a)).

Information is transmitted from the brain to the spinal cord through the brainstem. This part is also responsible for the control of the autonomous body functions (cardiac, respiratory, etc.) and certain motor functions. The cerebellum, located at the bottom of the head, is involved in the coordination of the body motions and in the time evaluation. Finally, the cerebrum is formed of two parts. Just above the brainstem, the diencephalon is in charge of the autonomous body functions 
and gets a neuroendocrine role (satiety, body temperature, sexuality, etc.). Then, the main part of the cerebrum is composed of the telencephalon, with its two cerebral hemispheres.

The right and left hemispheres are symmetrical. Their surface is covered by the cerebral convolutions (lat. gyri), surrounded by furrows (lat. sulci). In addition, the hemispheres tissues are organized in two layers. The outer one is the grey matter, also called cerebral cortex, constituted of neurons. The white matter, formed of axons, is located under the grey one.

The CNS (i.e. the encephalon and the spinal cord) is enclosed by tree membranes, called the meninges. Among them, the dura mater, hard and fibrous, is stuck to the bone (respectively the skull and the vertebra), ensuring its mechanical protection. Furthermore, two dividing walls are formed by folds of the dura mater: the falx cerebri, separating both hemispheres, and the tentorium cerebelli, between the brain and the cerebellum.

\subsection{Ventricular system and cerebrospinal fluid}

The ventricular system, located in the middle of the telencephalon, is mainly composed of four cavities: the two lateral (left and right), third and fourth ventricles (see Figure 7.1 (b)). The Cerebrospinal Fluid (CSF), which immerses all the CNS, is produced and dispatched by these ventricles (mostly the lateral ones). This liquid is composed of $99 \%$ water. Its total volume is approximately 120 to $150 \mathrm{~mL}$ for an adult and renewed three to four times per day. Several roles are handled by the CSF. First, it protects the brain against infections (thanks to its biochemical composition) and mechanically against impacts. Next, hormones and biological agents are transmitted to the different parts of the brain through this fluid.

\subsection{Brain vascularization}

As shown in Figure 7.2 (a), blood is supplied to the brain through three arteries: the two internal carotid arteries (left and right) and the basilar artery. Inside the cranial cavity, these three arteries are divided as follows :

- two arteries, the anterior cerebral and middle cerebral, are formed by each internal carotid

- the basilar artery is split into two posterior cerebral arteries (left and right)

Each hemisphere is then irrigated by three arteries: an anterior, a middle and a posterior one. In addition, these six major arteries are linked by the arterial circle of Willis (Figure 7.2(b)), located at the base of the brain. This leads to a partial overlap of the irrigating fields of each artery, allowing a better repartition of the blood flow for example in case of stroke. 


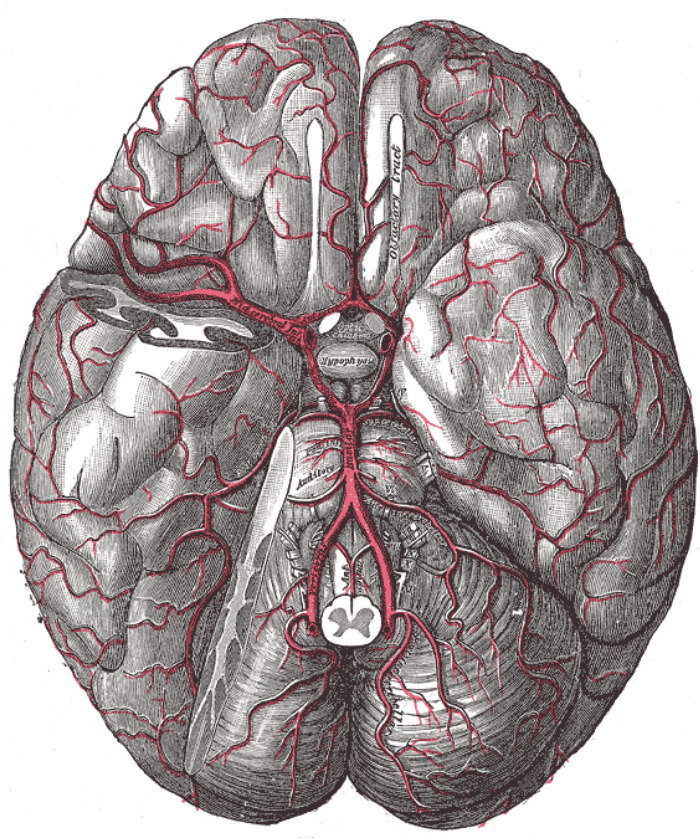

(a) Overview

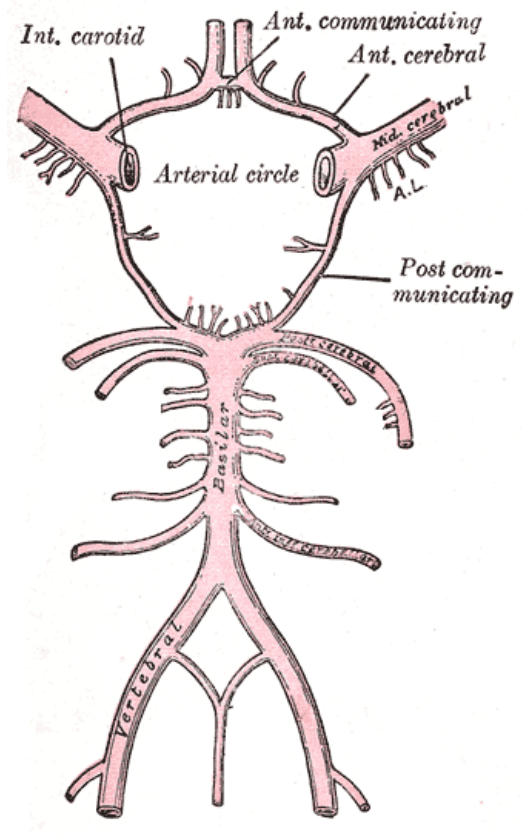

(b) Arterial circle of Willis

Figure 7.2: Main cerebral arteries (illustrations from 'Gray's anatomy', H. Gray, 1918)

\section{Brain Finite Element Models in the Literature}

In this section, an overview of the main brain modeling works are presented. A survey dealing with the biomechanical properties, with both the constitutive laws and their parameters, is introduced before the impacts of boundary conditions are discussed. Finally, main finite element brain models are reviewed in their applicative context.

\subsection{Mechanical properties of brain tissues}

Brain mechanical properties have been widely studied. While an analyze of a cranial trauma and brain motions was proposed by Pudenz and Shelden (1946) using direct observation of a monkey brain in 1946, one of the first surveys focusing on the biomechanical properties of the organ dates back to the 70's (Ommaya (1968)). Nowadays, improved methods have been developed in order to determine quantitative and accurate soft tissue characterization, allowing complex mathematical models. On the one hand, these biomechanical properties can be determined using rheological studies on animal or human brains. Experimental measurements (obtained by compression, tension or suction of the tissues) are then correlated with the numerical simulation of a FE model. On the other hand, biomechanical parameters can be estimated based on medical images such as Magnetic Resonance Imaging (MRI) or MR Elastography (MRE).

The brain biomechanical parameters found with both approaches are presented in the two following subsections. Even if a non-linear behavior is often highlighted by rheological experiments, 
the equivalent Young's moduli $E^{*}$ at small deformations are given when possible for comparison. In addition, when using elastography acquisitions, only the shear modulus $\mu$ is provided. However, it is linked to the Poisson's ratio $\nu$ and Young's modulus $E$ by $E=2 \mu(1+\nu)$. For indication, corresponding Young's moduli $E^{*}$ are then computed for $\nu=0.45$.

\subsubsection{Rheological experiments}

A bi-phasic poro-elastic model, validated using in vivo indentations on porcine brains, is proposed by Paulsen et al. (1999) and Miga et al. (2000). The brain is considered as a sponge-like material and modeled as a porous solid tissue with interstitial fluid. A linear elastic law is used for the solid tissues, with parameters set to $E=2.1 \mathrm{kPa}$ and $\nu=0.45$, and the pore fluid is considered as incompressible.

Also based on in vivo experiments on swine brains, Miller and his group conclude that:

- the relation between strain and constraints are strongly non-linear

- the stiffness of the brain tissues is higher in compression than in tension

- the behavior depends on the velocity of the loads (viscoelasticity)

According to these observations, an Ogden-like hyper-viscoelastic model with a Prony-series relaxation modulus is proposed for very soft tissues (Miller and Chinzei, 2002):

$$
\begin{gathered}
W=\frac{2}{\alpha^{2}} \int_{0}^{t}\left[\mu(t-\tau) \frac{\mathrm{d}}{\mathrm{d} \tau}\left(\lambda_{1}^{\alpha}+\lambda_{2}^{\alpha}+\lambda_{3}^{\alpha}-3\right)\right] \mathrm{d} \tau \\
\mu=\mu_{0}\left[1-\sum_{k=1}^{n} g_{k}\left(1-e^{-t / \tau_{k}}\right)\right]
\end{gathered}
$$

where $W$ is the strain energy and $\lambda_{i}$ the principal stretches. While $\alpha$ is a material coefficient without physical meaning, $\mu_{0}$ corresponds to the instantaneous shear modulus in the undeformed state. These parameters are respectively identified as -4.7 and $0.842 \mathrm{kPa}$ (corresponding to an equivalent Young modulus at small deformations $E^{*}=2.442 \mathrm{kPa}$ for $\nu=0.45$ ). Finally, $t$ and $\tau$ denote time, with $g_{k}$ a constant defined for characteristic relaxation times $\tau_{k}$. In the above model, the brain tissues are assumed to be incompressible and isotropic at the scale of the organ $(\mathrm{cm})$. This hypothesis is however nuanced by (Prange and Margulies, 2002) who showed some directional variations in the mechanical behavior at a finer level of modeling $(\mathrm{mm})$.

To our knowledge, the only rheological experiment on an in vivo human brain is presented by Schiavone et al. (2009). The biomechanical properties of the organ are measured intra-operatively with a light aspiration device, just before the beginning of tumor ablation procedure. To better fit 
the experimental results, a modified 2-term Mooney-Rivlin law is proposed:

$$
W=c_{10}\left(I_{1}-3\right)+c_{30}\left(I_{1}-3\right)^{3}
$$

where $I_{1}$ is the first invariant of the right Cauchy-Green strain tensor $C$ with $I_{1}=\operatorname{trace}(C)$. The two material constants $c_{10}$ and $c_{30}$ are respectively identified as $0.24 \mathrm{kPa}$ (equivalent Young's modulus at small deformations: $E^{*}=1.44 \mathrm{kPa}$ ) and $3.42 \mathrm{kPa}$. Finally, the brain tissues are considered as nearly incompressible with Poisson's ratio $\nu=0.45$.

Experiments on bovine brain tissues under uniaxial compression are presented by Laksari et al. (2012). The material is assumed to be homogeneous (white matter only), isotropic and almost incompressible. This last hypothesis then implies that the soft tissues behave differently in shear than in bulk and the strain energy can be written as:

$$
W=W_{i s o}+W_{v o l}
$$

A 3-term Mooney-Rivlin law and a 2-parameter Ogden model are respectively proposed for the isochoric and volumetric parts of the deformations:

$$
\begin{gathered}
W_{i s o}=c_{10}\left(\bar{I}_{1}-3\right)+c_{01}\left(\bar{I}_{2}-3\right)+c_{11}\left(\bar{I}_{1}-3\right)\left(\bar{I}_{2}-3\right) \\
W_{v o l}=\frac{K}{\alpha^{2}}\left[\alpha \ln (J)+J^{-\alpha}-1\right]
\end{gathered}
$$

where $\bar{I}_{i}$ are the invariants of $\bar{C}=J^{-2 / 3} C$ and $J=\operatorname{det}(F)=\lambda^{1-2 \nu}$ with $F$ being the deformation gradient and $\lambda$ the stretch ratio in the direction of compression. The material parameters are then derived as follows: $c_{10}=-1.34 \mathrm{kPa}, c_{01}=1.83 \mathrm{kPa}, c_{11}=0.29 \mathrm{kPa}, \alpha=100, K=46 \mathrm{kPa}$ and $\nu=0.49$. The authors argue that since a negative $c_{10}$ is found in this study and would have a non physical meaning for a 2-term Mooney Rivlin model, at least 3 parameters are required for $W_{\text {iso }}$ to accurately capture the material behavior.

Finally, stiffness differences are pointed out by Kaster et al. (2011) for white and grey matter. To do so, experiments using indentations of ex vivo porcine brain slices are presented. Several commonly used models (Polynomial, Yeoh, Arruda-Boyce and Ogden models) are fit with the experimental data, providing sets of hyperelastic parameters. The equivalent Young's moduli are finally computed as $1.787 \pm 0.186 \mathrm{kPa}$ and $1.195 \pm 0.157 \mathrm{kPa}$ respectively for the white and grey matter. Very similar results are also shown by Budday et al. (2015) using indentations of ex vivo bovine slices. The Young's modulus is then directly computed based on the experiments (i.e. no 
biomechanical model is run to fit the observations) and found on average equal to $1.895 \pm 0.592$ $\mathrm{kPa}$ and $1.389 \pm 0.289 \mathrm{kPa}$ respectively for the white and grey matter.

\subsubsection{Imaging methods}

The stiffness of brain tissues could be determined using MRE acquisitions. Muthupillai et al. (1995) proposed to estimate material's shear modulus from the harmonic shear wave velocity. Since this method is non-invasive, it is performed on in vivo human brains to measure the white and grey matter stiffness. Results with corresponding publications are reported in Table 7.1 . In addition, equivalent Young's moduli $E^{*}$ are computed for $\nu=0.45$. A mean shear modulus (with no differentiation of white and grey matter) equal to $3.5 \mathrm{kPa}\left(E^{*}=10.15 \mathrm{kPa}\right)$ is also proposed by Hamhaber et al. (2007). Values reported in this paragraph then appear significantly higher than the ones found with rheological experiments (see Table 7.2). However, the measured $\mu$ decreases with the shear wave frequency (Chatelin et al. 2010). For finite element simulations, a static Young's modulus is required, that would correspond to a null excitation frequency to avoid any viscosity effects. Therefore, Young's moduli reported above probably overestimate this static value.

Table 7.1: Shear moduli (in $\mathrm{kPa}$ ) found using MRE and indicative Young's moduli (in $\mathrm{kPa}$ ) computed for $\nu=0.45$

\begin{tabular}{|c|c|c|c|c|}
\hline \multirow{2}{*}{ Publication } & \multicolumn{2}{|c|}{ White matter } & \multicolumn{2}{|c|}{ Grey matter } \\
\hline & $\mu$ & $E^{*}$ & $\mu$ & $E^{*}$ \\
\hline Kruse et al. (1999) & 14.6 & 42.34 & 6.43 & 18.65 \\
\hline Uffmann et al. $(\overline{2004})$ & $15.2 \pm 1.4$ & $44.08 \pm 4.06$ & $12.9 \pm 0.9$ & $37.41 \pm 2.61$ \\
\hline McCracken et al. $(2005)$ & $10.7 \pm 1.4$ & $31.03 \pm 4.06$ & $5.3 \pm 1.3$ & $15.37 \pm 3.77$ \\
\hline Green et al. $(2006)$ & 2.1 & 6.09 & 2.8 & 8.12 \\
\hline Kruse et al. $(2008)$ & 13.6 & 39.44 & 5.22 & 15.14 \\
\hline
\end{tabular}

Relying on classic MR data, a new non-invasive method for the determination of the human brain properties is proposed by Soza et al. (2004). Pre- and intraoperative MRI are first elastically registered using a biomechanical model. Next, $E$ and $\nu$ are estimated based on the mutual information of the registered pre- and intraoperative MRI. This experiment is presented for low (9952 elements) and high (123496 elements) resolution FE models with respective reported results $E_{\text {low }}=8.863 \mathrm{kPa}, \nu_{\text {low }}=0.452$ and $E_{\text {high }}=8.196 \mathrm{kPa}, \nu_{\text {high }}=0.461$.

\subsubsection{Conclusion}

A large number of studies dealing with the determination of brain soft tissues constitutive law and biomechanical parameters can be found in the literature. From the previous overview, the main features are :

- the mechanical behavior of the brain is highly non-linear 
- the brain tissues are quasi incompressible (Poisson's ratio $\nu \geq 0.45$ ) and their stiffness is very low (Young's modulus at small deformations $E$ is about few $\mathrm{kPa}$ )

- stiffness differences exist between white and grey matter

As seen in Table 7.2 important differences are also pointed out and a consensus on an accurate characterization of the brain tissues is still difficult to obtain.

These differences could be explained by the diversity of the experimental protocols (rheologicalor image-based) and conditions (in vivo, in vitro or ex vivo on human or animal brains). A survey focusing on the influence of these testing methods and protocols is presented by Hrapko et al. (2008). In addition, while the in vitro and in vivo experimental protocols are reviewed and compared by Chatelin et al. (2010), only the imaging-based methods are considered by Bayly et al. (2012).

The dispersion of the reported values might also be due to inter subject differences. An MRE study presented by (Sack et al. 2009), including 55 human volunteers (23 females) aged from 18 to 88 years, showed significant brain stiffness differences according to the sex and age of the subject. While female brains are on average $9 \%$ stiffer than male ones, a liquefaction of the organ with age is observed with a decrease of the shear modulus of $0.8 \%$ per year. Similarly, Chatelin et al. (2012) showed that adult brains are on average 3 to 4 times stiffer than children (aged from 5 to 22 months) ones.

In addition, all values reported in the above paragraphs are dealing with healthy tissues. However, considering patient suffering from brain disorder, the mechanical behavior could vary depending on the pathology and its treatments. For example, stiffness differences between tumor and healthy surrounding white matter tissues are highlighted by Xu et al. (2007) using MRE. Furthermore, stiffer brain tissues are often reported by neurosurgeons when patients are treated with radiotherapy. Finally, a complete review of the brain mechanics is proposed by Goriely et al. (2015), pointing out the challenges related to neurodevelopment and various brain disorders.

\subsection{Boundary conditions and loading}

While getting an accurate description of the mechanical behavior is essential, boundary conditions and loads imposed on the organ are at least as important in the FE modeling. On the one hand, boundary conditions can be defined according to the anatomy (contacts with the skull/dura mater, modeling of the falx cerebri and tentorium cerebelli, etc.). On the other hand, loads are specific boundary conditions imposed through displacements or forces, depending on the interactions with the organ during simulation.

Miller and his group investigated on the sensitivity to the mechanical properties when loadings are imposed through displacements. To do so, such loads are applied by Wittek et al. (2009) to 
an FE brain model simulated according to three different constitutive laws: a hyperviscoelastic, a hyperelastic and a linear elastic law. No significant differences are then showed for the solution in displacements. However, important ones are pointed out when the simulation is realized under the hypothesis of small displacements. In this specific context, the authors therefore advise to use linear elastic constitutive law with geometrically non-linear analysis in order to get a sufficient accuracy while sparing computation time. In addition, a similar result is presented by Miller and Lu (2013). Since the solution in displacements is weakly sensitive to the chosen biomechanical properties, the neo-Hookean constitutive law, being the simplest non-linear model, is recommended to simulate brain deformations under imposed displacements.

Conversely, Valencia et al. (2012) proposed to study the impact of the biomechanical properties when a pressure is applied on the cortical surface. Simulations are performed for elastic and various hyperelastic constitutive laws (neo-Hookean, 1st, 2nd and 3rd Ogden models and Mooney-Rivlin models with 2 and 5 terms). Resulting displacements and stresses are then compared reporting important differences, especially for stress results.

For the FE modeling, the choice of the brain biomechanical properties is thus closely linked to the simulation purpose. It is often a trade-off between high mechanical behavior accuracy and execution times requirements, depending on boundary conditions and loads imposed on the organ. Indeed, according to Bilston (2011), "it's unrealistic to expect that one constitutive model will fit all circumstances".

Table 7.2: Summary of the constitutive laws and parameters. All stiffness parameters $\left(E, \mu, K, c_{i}\right.$ and $\left.c_{i}\right)$ are given in $\mathrm{kPa}$. For hyper elastic laws and elastography studies, equivalent Young's moduli $E^{*}$ are computed for comparison.

\begin{tabular}{|c|c|c|c|}
\hline Publication & Constitutive law & Parameters & Context \\
\hline $\begin{array}{l}\text { Paulsen et al. } \\
\begin{array}{l}(1999) \text { and } \\
\text { et al. }(2000)\end{array}\end{array}$ & Bi-phasic poro-elastic model & $E=2.1, \nu=0.45$ & \multirow{6}{*}{$\begin{array}{l}\text { Rheological } \\
\text { experiments }\end{array}$} \\
\hline $\begin{array}{l}\text { Miller and Chinzei } \\
(2002)\end{array}$ & $\begin{array}{l}\text { Ogden-like model with a re- } \\
\text { laxation modulus }\end{array}$ & $\begin{array}{l}\alpha=-4.7, \mu_{0}=0.842 \\
\left(E^{*}=2.442 \text { for } \nu=0.45\right. \\
\text { at undeformed state })\end{array}$ & \\
\hline $\begin{array}{l}\text { Schiavone } \text { et } \text { al. } \\
(2009) \\
\end{array}$ & $\begin{array}{l}\text { Modified 2-term } \text { Mooney- } \\
\text { Rivlin model }\end{array}$ & $\begin{array}{l}c_{10}=0.24, c_{30}=3.42 \\
\nu=0.45\left(E^{*}=1.44\right)\end{array}$ & \\
\hline Kaster et al. $(2011)$ & $\begin{array}{l}\text { Polynomial, Yeoh, Ogden and } \\
\text { Arruda-Boyce models }\end{array}$ & $\begin{array}{l}E_{\text {white }}^{*}=1.787 \pm 0.186 \\
E_{\text {grey }}^{*}=1.195 \pm 0.157\end{array}$ & \\
\hline $\begin{array}{lll}\text { Laksari } & \text { et } & \text { al. } \\
(2012) & & \end{array}$ & $\begin{array}{l}\text { 3-term Mooney-Rivlin and } \\
\text { 2-parameter Ogden models } \\
\text { (resp. for the isochoric } \\
\text { and volumetric deformations } \\
\text { parts) }\end{array}$ & $\begin{array}{l}c_{10}=-1.34, c_{01}=1.83 \\
c_{11}=0.29, \alpha=100, K= \\
46 \text { and } \nu=0.49\end{array}$ & \\
\hline $\begin{array}{lll}\text { Budday } & \text { et } & \text { al. } \\
(2015) & & \\
\end{array}$ & - & $\begin{array}{l}E_{\text {white }}=1.895 \pm 0.592 \\
E_{\text {grey }}=1.389 \pm 0.289\end{array}$ & \\
\hline $\begin{array}{l}\text { Hamhaber et al. } \\
(2007)\end{array}$ & - & $\mu=3.5\left(E^{*}=10.15\right)$ & \multirow[b]{2}{*}{$\begin{array}{l}\text { Estimation } \\
\text { using MR } \\
\text { Elastogra- } \\
\text { phy }\left(E^{*}\right. \\
\text { computed } \\
\text { for } \nu=0.45)\end{array}$} \\
\hline & 9 & & \\
\hline
\end{tabular}


Table 7.2 - continued from previous page

\begin{tabular}{|c|c|c|c|}
\hline Publication & Constitutive law & Parameters & Context \\
\hline 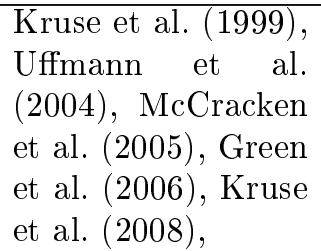 & - & $\begin{array}{llr}\mu_{\text {white }} & \in & {[2.1 ; 15.2]} \\
\left(E_{\text {white }}^{*} \in\right. & [6 ; 44]), \\
\mu_{\text {grey }} \in & {[2.8 ; 12.9]} \\
\left(E_{\text {grey }}^{*} \in\right. & [8 ; 38]), \quad \text { see } \\
\text { Table } 7.1 & \text { for details }\end{array}$ & \\
\hline Soza et al. $(2004)$ & Linear poro-elastic model & $\begin{array}{l}E \quad \in[8.2 ; 8.85], \quad \nu \quad \in \\
{[0.4552 ; 0.461]}\end{array}$ & $\begin{array}{l}\text { Estimation } \\
\text { using MRI } \\
\text { registration }\end{array}$ \\
\hline Clatz et al. $(2005)$ & Linear elastic law & $\begin{array}{l}E=0.694, \nu=0.45 \\
E_{\text {ventricles }}=0.01, \\
\nu_{\text {ventricles }}=0.05\end{array}$ & \multirow{9}{*}{$\begin{array}{l}\text { Brain-shift } \\
\text { compensa- } \\
\text { tion for } \\
\text { tumor } \\
\text { ablation } \\
\text { procedures }\end{array}$} \\
\hline Wittek et al. $(\overline{2009})$ & Linear elastic law & $E=2.5, \nu=0.49$ & \\
\hline $\begin{array}{l}\text { Dumpuri et al. } \\
\begin{array}{l}(2006) \text { and } \\
\text { et al. }(2011)\end{array}\end{array}$ & $\begin{array}{l}\text { Bi-phasic poro-elastic model } \\
\text { (Paulsen et al. }(1999))\end{array}$ & $E=2.1, \nu=0.45$ & \\
\hline $\begin{array}{l}\text { Vigneron et al. } \\
(2012)\end{array}$ & Linear elastic law & $E=3, \nu=0.45$ & \\
\hline $\begin{array}{l}\text { De Lorenzo et al. } \\
(2012)\end{array}$ & Linear elastic law & $E=66.7, \nu=0.48$ & \\
\hline Bucki et al. $(2012)$ & Linear elastic law & $\begin{array}{l}E=0.694, \nu=0.4, \\
E_{\text {ventricles }}=0.01, \\
\nu_{\text {ventricles }}=0.05\end{array}$ & \\
\hline \begin{tabular}{|lll} 
Miller & and & Lu \\
$(2013)$ & &
\end{tabular} & Neo-Hookean law & $E^{*}=3, E_{\text {tumor }}^{*}=9$ & \\
\hline $\begin{array}{l}\text { Mohammadi et al. } \\
(2015)\end{array}$ & Linear elastic law & $\begin{array}{l}E=0.700, \nu=0.42, \\
E_{\text {ventricles }}=0.015 \\
\nu_{\text {ventricles }}=0.05\end{array}$ & \\
\hline$\overline{\text { Morin et al. }}(\overline{2016})$ & $\begin{array}{l}\text { Linear elastic law with co- } \\
\text { rotational approach }\end{array}$ & $\begin{array}{l}E=1.5, \nu=0.45, \\
E_{\text {tumor }}=10, \nu_{\text {tumor }}= \\
0.45\end{array}$ & \\
\hline Hu et al. (2007) & Zener model & - & $\begin{array}{l}\text { Brain-shift } \\
\text { prediction }\end{array}$ \\
\hline Clatz et al. $(2003)$ & Linear elastic law & $E=2, \nu=0.45$ & \multirow{2}{*}{$\begin{array}{l}\text { Brain-shift } \\
\text { (Parkinson } \\
\text { disease) }\end{array}$} \\
\hline Bilger et al. $(2011)$ & $\begin{array}{l}\text { St Venant Kirchhoff law with } \\
\text { co-rotational approach }\end{array}$ & $E=6, \nu=0.45$ & \\
\hline $\begin{array}{l}\text { Takizawa et } \text { al. } \\
(1994)\end{array}$ & Linear elastic law & $\begin{array}{l}E_{C S F}=1, E_{\text {falx }}=100, \\
E_{\text {white }}=4, E_{\text {grey }}=8, \nu= \\
0.47\end{array}$ & \multirow{4}{*}{$\begin{array}{l}\text { Pathological } \\
\text { region } \\
\text { growing }\end{array}$} \\
\hline $\begin{array}{l}\text { Kyriacou et } \\
(1999)\end{array}$ & Neo-Hookean law & $\begin{array}{l}c_{10, \text { white }}=\quad 3 \\
\left(E_{\text {white }}^{*}=18\right), \\
c_{10, \text { grey }}=c_{10, \text { tumor }}=30 \\
\left(E_{\text {grey }}^{*}=E_{\text {tumor }}^{*}=180\right)\end{array}$ & \\
\hline $\begin{array}{lll}\text { Prastawa et al. } \\
(2009)\end{array}$ & $\begin{array}{l}\text { Linearized homogenous ver- } \\
\text { sion of Miller et al. }(2002)\end{array}$ & $\begin{array}{l}E_{\text {brain }}=0.694, E_{\text {falx }}= \\
200, \nu=0.4\end{array}$ & \\
\hline Yousefi et al. $(2013)$ & Linear elastic law & $\begin{array}{l}E=3 \pm 0.25, \nu=0.45 \pm \\
0.145\end{array}$ & \\
\hline $\begin{array}{l}\text { Castellano-Smith } \\
\text { et al. }(2003)\end{array}$ & Linear elastic law & $\begin{array}{l}E_{\text {white }}=4, E_{\text {grey }}=8, \nu= \\
0.495\end{array}$ & \multirow[b]{2}{*}{$\begin{array}{l}\text { Simulation } \\
\text { of abnormal } \\
\text { developments }\end{array}$} \\
\hline $\begin{array}{lll}\text { Budday et al. } \\
(2014)\end{array}$ & Neo-Hookean law & $\begin{array}{l}\mu_{\text {cortex }}=3 * \mu_{\text {subcortex }}= \\
3.159, \quad \nu \quad=0.458 \\
\left(E_{\text {cortex }}^{*}=3 * E_{\text {subcortex }}^{*}=\right. \\
9.21)\end{array}$ & \\
\hline
\end{tabular}


Table 7.2 - continued from previous page

\begin{tabular}{|l|l|l|l|}
\hline \multicolumn{1}{|c|}{ Publication } & \multicolumn{1}{|c|}{ Constitutive law } & \multicolumn{1}{c|}{ Parameters } & \multicolumn{1}{c|}{ Context } \\
\hline $\begin{array}{l}\text { Dequidt et al. } \\
(2015)\end{array}$ & $\begin{array}{l}\text { Linear elastic law with co- } \\
\text { rotational approach }\end{array}$ & $\begin{array}{l}\text { Brain } \\
\text { surgery } \\
\text { simulator }\end{array}$ \\
\hline Sase et al. $(2015)$ & $\begin{array}{l}\text { Linear elastic law with co- } \\
\text { rotational approach }\end{array}$ & $E=1, \nu=0.4$ & 0.45 \\
\hline
\end{tabular}

\subsection{Biomechanical models}

In the next paragraphs, brain models are presented in their applicative framework and summarized in Table 7.2. The chosen biomechanical properties as well as the boundary conditions are highlighted.

\subsubsection{Brain-shift preoperative prediction and intraoperative compensation for wide opening surgery}

For tumor ablation procedure, accurate localization of the target is essential. Planning and guidance are then based on MR images acquired prior to surgery. However, the intraoperative deformation of the brain soft tissues, called brain-shift, affects this localization (Gerard et al. 2017).

For the intraoperative guidance, brain-shift compensation methods propose to register preoperative MR images with data (images or surface data) acquired during surgery. In order to capture the current deformations of the brain, Vigneron et al. (2012) proposed to perform intraoperative MR acquisitions. Displacements are imposed over a biomechanical model simulated using linear elastic constitutive law $(E=3 \mathrm{kPa}$ and $\nu=0.45)$ to register the pre- and intraoperative MRIs. Extended FE method is used to represent discontinuities between two consecutive intraoperative MR acquisitions. In addition, an heterogeneous brain model is presented by Clatz et al. (2005). Properties of the parenchyma are assigned to $E=0.694 \mathrm{kPa}$ and $\nu=0.45 \mathrm{by}$. In order to simulate the CSF loss during the registration process, the ventricles parameters are fixed to $E=0.01 \mathrm{kPa}$ and $\nu=0.05$. Similar heterogeneous brain models are also proposed by Bucki et al. (2012) and Mohammadi et al. (2015). Displacements are then imposed to register the vascular trees extracted from preoperative MR and intraoperative ultrasound (US) acquisitions.

Other brain-shift compensation methods track the exposed cortical surface using laser-range scanners or stereo-cameras. De Lorenzo et al. (2012) proposed to impose displacements on the surface of a biomechanical model to recover the full deformations of the brain. This model is simulated according to a linear elastic law, with parameters $E=66.7 \mathrm{kPa}$ and $\nu=0.48$. Contacts with the skull are accounted for and FE surface nodes at the base of the brain in the inferior occipital lobes (i.e. close to the tentorium cerebelli and far from the craniotomy region) are fixed. Similarly, Sun et al. (2014) used a biomechanical model to preoperatively build an atlas mesh of the 
soft tissues deformations. During surgery, a minimization problem is solved in order to compare the acquired cortical surface data to the precomputed atlas meshes. The constitutive law proposed by Paulsen et al. (1999) with parameters $E=2.1 \mathrm{kPa}$ and $\nu=0.45$ is used for the simulation of the soft tissues. In addition, an automatic method for the generation of the boundary conditions is presented by Dumpuri et al. (2006). The brain surface is then split into three parts. While the upper part, close to the craniotomy, is stress-free, the lower cerebellum part is fixed and sliding contacts are applied between the remaining brain surface and cranial wall. Furthermore, the same slip boundary conditions are assigned by Chen et al. (2011) to the falx cerebri and tentorium cerebelli.

A biomechanical model predicting gravity-induced brain-shift after opening of the dura mater is proposed by Hu et al. (2007) for the preoperative planning. The white and grey matters, ventricles, dura mater, falx cerebri, tentorium cerebelli, brainstem and cerebellum are accounted for. While isotropic elastic shell elements with high stiffness are used to represent the membranes $(E=31.5$ $\mathrm{MPa}$ and $\nu=0.45$ ), the white and grey matters, brainstem and cerebellum are simulated using a Zener model which is a representation using springs of a linear viscoelastic material (see table 1 in their publication for parameters). Finally, few hours are needed for the resolution of this complex biomechanical model.

\subsubsection{Brain-shift preoperative prediction for Parkinson's disease procedure}

Brain disorders, such as Parkinson's disease, can be treated using Deep Brain Stimulation. During this surgical procedure, electrodes are deeply implanted into the brain though a small opening of the skull to stimulate functionally deficient areas with electrical impulses. The targeted position and trajectory of the electrodes are usually computed on preoperative MRI. However, the brain-shift due to the CSF leakage affects the accuracy of the procedure. Clatz et al. (2003) proposed to predict these soft tissues deformations so that the electrodes could be well positioned. For this purpose, the brain is simulated with a linear elastic law $(E=2 \mathrm{kPa}$ and $\nu=0.45)$ and the CSF is modeled as a liquid applying pressure on the surface of the organ. A similar CSF modeling technique is followed by Bilger et al. (2011). However, the brain simulation is performed based on a St Venant-Kirchhoff constitutive law, with parameters $E=6 \mathrm{kPa}$ and $\nu=0.45$, and a corotational approach (see paragraph 4.2.2 is used. In addition, each hemisphere is modeled independently with a contact region between them in order to capture the behavior of the falx cerebri.

\subsubsection{Simulation of pathological region growing}

The growing of a pathological region, such as an edema, hematoma or meningioma, leads to soft tissues deformations and then residual stress within the organ. Brain dysfunctions might 
therefore appear, depending on this stress level and localization. For prevention and decision support, Takizawa et al. (1994) proposed to study the stress distribution caused by an intracerebral hematoma. A 2D model of a single hemisphere is simulated following a linear elastic law. The Young's moduli for the CSF, falx cerebri and white and grey matters are respectively set to $E_{C S F}=$ $1 \mathrm{kPa}, E_{\text {falx }}=100 \mathrm{kPa}, E_{\text {white }}=4 \mathrm{kPa}$ and $E_{\text {grey }}=8 \mathrm{kPa}$. The lateral ventricle is assumed to be empty in order to account for the CSF leakage. Finally, the brain surface is considered attached to the skull and fixed. Another 2D model is presented by Kyriacou et al. (1999) to simulate a tumor growth. The neo-Hookean constitutive law is used with parameters $c_{10, \text { white }}=3 \mathrm{kPa}\left(E_{\text {white }}^{*}=18\right.$ $\mathrm{kPa})$ and $c_{10, \text { grey }}=c_{10, \text { tumor }}=30 \mathrm{kPa}\left(E_{\text {grey }}^{*}=E_{\text {tumor }}^{*}=180 \mathrm{kPa}\right)$. In addition, the dura mater, falx cerebri and tentorium cerebelli are assumed to be rigid.

More recently, 3D FE models have also been proposed. In order to evaluate segmentation algorithms, Prastawa et al. (2009) simulated the growing of a brain tumor and edema within healthy MRIs. A linearized homogeneous version of the model proposed by Miller et al. (2002) is used. While the Poisson's ratio is uniformly set to $\nu=0.4$, the Young's moduli for the soft tissues and the falx cerebri are respectively fixed to $E_{\text {brain }}=0.694$ and $E_{\text {falx }}=200 \mathrm{kPa}$. In addition, sliding contacts are modeled between the brain and skull. More specifically, the growing of a meningioma is studied by Yousefi et al. (2013). This king of tumor is located in the meninges and brain tissues are compressed from the cortical surface when it grows. The brain is simulated according to a linear constitutive law. However, their parameters are optimized in order to better fit the brain morphology and tumor size of each patient. Initially set to $E=3 \mathrm{kPa}$ and $\nu=0.45$, the optimized values found over 7 patients are $E=3 \pm 0.25 \mathrm{kPa}$ and $\nu=0.45 \pm 0.145$.

\subsubsection{Prediction of abnormal development of the brain}

Brain biomechanical models have been proposed in order to understand or predict abnormal developments. Budday et al. (2014) showed that malformation diseases, such as lissencephaly, polymicrogyria or schizophrenia, originate from a discrepancy between cortical and subcortical growth. For that purpose, a brain FE model is used to simulate the development of neonatal soft tissues. These tissues are modeled as Neo-Hookean elastic with parameters three times stiffer for the cortex than for the subcortex $\left(E_{\text {cortex }}^{*}=3 * E_{\text {subcortex }}^{*}=9.21 \mathrm{kPa}\right.$ and $\left.\nu=0.458\right)$.

Finally, a cerebral atrophy is observed in some dementia diseases such as Alzheimer's disease. A biomechanical simulation of brain atrophy is proposed by Castellano-Smith et al. (2003). The choice of a small displacement linear elastic model is justified by the authors by the small size of an atrophy regarding the total brain volume. The Young's moduli for the white and grey matters are respectively set to $E_{\text {white }}=4$ and $E_{\text {grey }}=8 \mathrm{kPa}$, with $\nu=0.495$. 


\subsubsection{Brain surgery simulator}

Brain surgery simulators are aimed to train students to practice surgical procedures. Highrate responses (especially when haptic feedback is provided) but also physical accuracy are needed. However, even if free loads are imposed using some virtual surgical instruments, simulations are most of the time performed with linear constitutive laws in order to comply with the execution time requirements.

A brain surgery simulator with a linear elasticity, a rubber material and various levels of accuracy is presented by Vang Hausen and Vlhelm Larsen (1998). A region of interest around the surgical target is defined by the user and simulated using a dynamic FE model. The remaining part of the brain is modeled based on static equations. In order to reach in real-time requirements, the resolution of the global brain model is however very low (around thousand nodes).

More recently, the St Venant-Kirchhoff law is used for the brain surgery simulator proposed by Echegaray et al. (2014). The Total Lagrangian Explicit Dynamics (TLED) FE algorithm (Miller et al. (2007)) is elected for the resolution of the partial derivative equations. Geometric and material non linearities are handled by the TLED method which allows computing deformations for very soft tissues in real-time. Finally, both the vascular neurosurgery simulator proposed by Dequidt et al. (2015) and the opening brain fissure simulator presented in Sase et al. (2015) are simulated based on a linear elastic law solved with the corotational approach(see paragraph 4.2.2). Biomechanical parameters for these two simulators are respectively set to $E=2.1 \mathrm{kPa}, \nu=0.45$ and $E=1 \mathrm{kPa}$, $\nu=0.4$.

\subsection{Conclusion}

All constitutive laws and biomechanical parameters mentioned in this section are summarized in Table 7.2 according to their applicative context. Even if highly non linear behaviors are often reported by rheological studies, most brain biomechanical models are simulated with a linear elastic law. This choice is justified by authors based on rather small deformations observed, execution times requirements or kind of loads applied on the organ. With few exceptions, very low stiffness parameters are taken and brain soft tissues are considered nearly incompressible. In addition, depending on the applicative context, various brain anatomical elements (e.g. ventricles) or boundary conditions (e.g. contacts with the dura mater, falx cerebri or tentorium cerebelli) are accounted for. FE brain models should thus be carefully designed, regarding the targeted application.

\section{Biomechanical FE Model for Vessel-Based Brain-Shift Compensation}

In the next paragraphs, a FE model for intraoperative brain-shift compensation in the case of tumor ablation procedure is introduced. As stated in Section 3.3.1 that presents an overview 
of the literature from a biomechanical point of view, this deformation is major source of error in neuro-navigation systems. After briefly describing the existing works from a clinical perspective, our approach is detailed in the following subsections.

\subsection{Relative works}

In order to obtain information about the current soft tissues deformations, all brain-shift compensation methods rely on the acquisition of data during surgery. Various intraoperative imaging systems are used in the literature such as MR scanners, laser-range scanners, stereo cameras or US devices. However, the acquired data cannot be directly used for navigation: images are of poor quality compared to the preoperative MRI and surface data alone are not clinically relevant. Brain-shift compensation methods thus propose to register MR images acquired prior to surgery with intraoperative data.

Accurate and dense information are provided by intraoperative MRI (Hastreiter et al. 2004 Vigneron et al. 2012). However, the use of such MR scanner during surgical intervention is cumbersome. Indeed, the procedure is complex (transfer of the patient, specific tools due to the magnetic field) and heavily increases the operating time. In addition, MR scanners are expensive and therefore available in very few operating rooms. This intraoperative imaging technique is thus rarely used in clinical routine.

Laser-range scanners and stereo cameras provide information about the deformations of the exposed cortical surface. The methods relying on such acquisitions (De Lorenzo et al., 2012; Miga et al. 2015) therefore make the strong assumption that all the non-linear $3 \mathrm{D}$ deformations of the soft tissues can be extrapolated from the exposed brain surface. However, according to Wittek

et al. (2007), "the prediction accuracy improves when information about deformation of not only exposed (during craniotomy) but also unexposed parts of the brain surface is used when prescribing loading".

In the literature, methods relying on US acquisitions are also presented. Since US scanners are far more affordable than MR ones but also portable and compatible with other surgical equipments, they are available in most operating theaters. In addition, intraoperative US acquisitions do not necessitate any important changes in the surgical procedure.

On the one hand, brain soft tissues can be visualized using intraoperative B-mode imaging. Pureimage based registration methods are then proposed in the literature to register these images with the MRI acquired prior to surgery (Mercier et al. 2012, Fuerst et al. 2014, Rivaz and Collins, 2015). However, MR and US imaging rely on very different physical principles implying dissimilar image characteristics (intensity, noise, contrast, etc). Their registration is thus a challenging problem. 


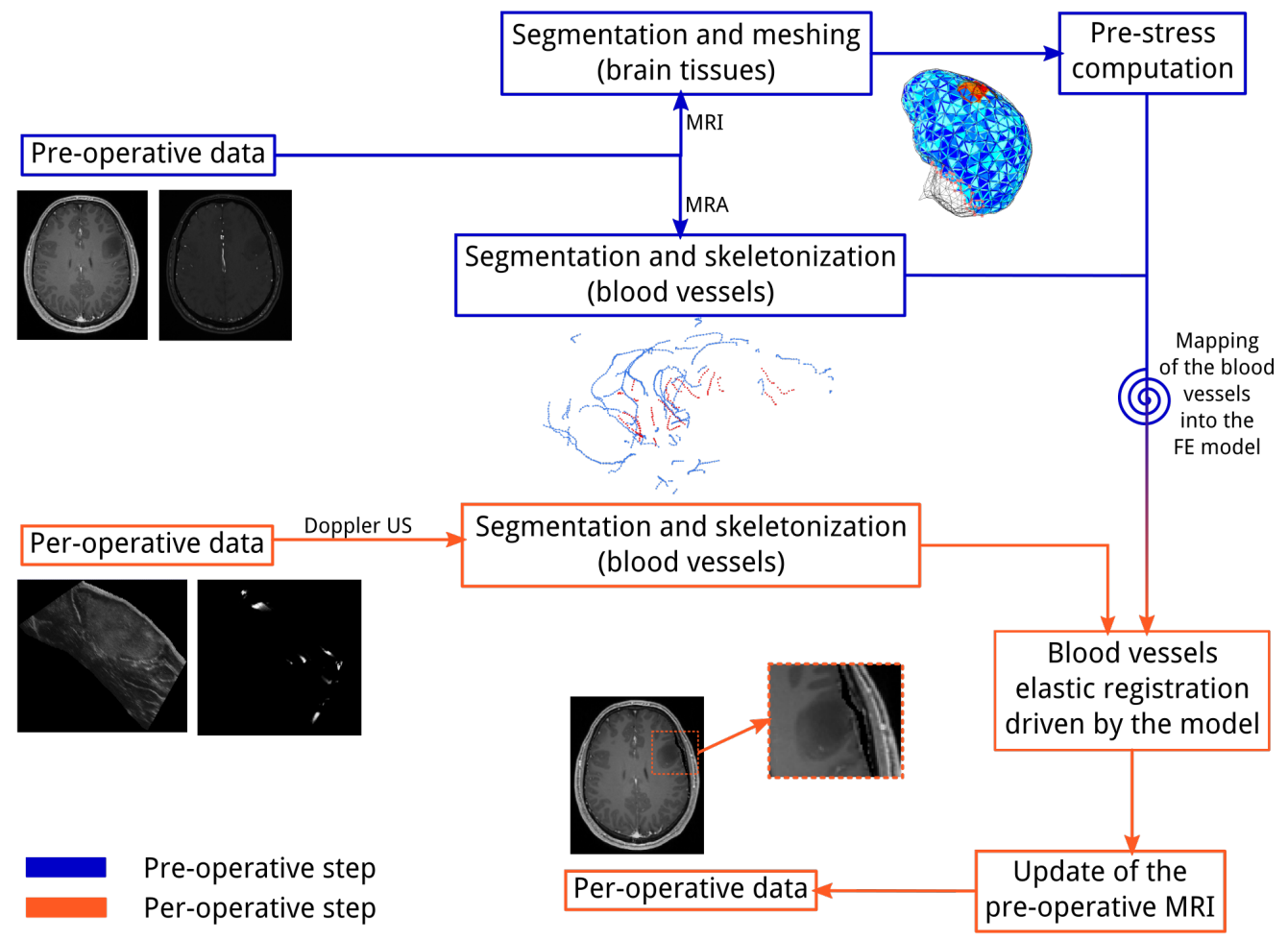

Figure 7.3: Pipeline of the brain-shift correction method

On the other hand, Doppler US imaging provides flow visualization. A rigid image-based method is proposed by Reinertsen et al. (2014) to register the vascular tree segmented from preoperative MR angiography (MRA) and intraoperative Doppler US. The efficiency of this vessel-based approach is validated on 7 patients. Model-based methods are also presented in the literature to register these vascular trees. Bucki et al. (2012) handled the registration using imposed displacements on blood vessels and provided evaluation over one clinical case. Finally, Mohammadi et al. (2015) proposed to combine tracking of the cortical surface with stereo cameras with Doppler US imaging. So far, their method is validated with a phantom study and animal brains only.

\subsection{Our approach}

Our method is based on the vessel-based approach described above (Reinertsen et al. , 2014), using intra-operative Power Doppler US acquisitions. The registration of the vascular tree is driven by a brain biomechanical model (Bucki et al. 2012). A global coherence could then be ensured by taking into account the tissues morphology and properties all over the organ and not only in the area covered by the US images. The method was presented in IPCAI'2016 (Morin et al. 2016), with improved results in comparison with a pure image-based registration (Reinertsen et al. 2014$)$ over a single clinical case. Moreover, the pre- and intraoperative processing steps remain compatible with a surgical process in term of execution time and user interactions. 
The pipeline of our brain-shift compensation method is detailed in Figure 7.3. An anatomical patient-specific brain model is built from preoperative MRI. Gravity-induced internal pre-stress is then computed (Morin et al. (2015)). In parallel, blood vessels are extracted from preoperative MRA and coupled with the biomechanical model. Intraoperatively, the deformed vascular tree is obtained from Power Doppler US acquisitions. The blood vessels registration is then driven by the FE model using Lagrangian Multipliers. While this process is described by Morin et al. (2016), following paragraphs focus on the brain modeling choices.

\subsubsection{Brain modeling and boundary conditions}

The cerebrum, cerebellum, brainstem and the tumor are segmented from preoperative MRI. The cerebrum and tumor are meshed, with a higher density of elements in the tumor area in order to better capture its deformations. The tentorium cerebelli is identified as the border between the cerebrum and cerebellum. Since this membrane is quite rigid, the nodes of the model located on the tentorium cerebelli are assigned to fixed Dirichlet conditions.

The dura mater surface is generated as the external surface of the brain FE mesh at the beginning of the simulation. As this membrane is stuck to the skull, it is fixed throughout the simulation. Sliding constraints are used, allowing the brain to move along the dura mater without any friction. Displacements in the normal direction inside the cranial cavity are allowed.

During the simulation, loads are imposed through displacements to register the vascular tree embedded within the model onto the US extracted data. Both these vessels loads and contacts between the brain and dura mater are handled using Lagrangian Multipliers, with an ICP-inspired method proposed by Courtecuisse et al. (2014).

\subsubsection{Constitutive law and biomechanical parameters}

As explained above, imposed displacements are used to drive the simulation. According to the conclusions of Wittek et al. (2009) and Miller and Lu (2013) in similar simulation cases, the solution in displacements is weakly sensitive to the chosen constitutive law and biomechanical properties (see Section 3.2.

Our simulation is then handled with a linear elastic law simulated with the corotational approach (Müller et al. 2002). First introduced in the field of computer graphics, this formulation is more and more used for medical simulations due to its low computational cost. The rotation of each element is evaluated independently, allowing better accuracy and thus a higher range of deformations. Forces and displacements are computed in the rotated system and finally transformed in the object one.

Following Schiavone et al. (2009) the Young's modulus and Poisson's ratio are respectively set to $E=1.5 \mathrm{kPa}$ and $\nu=0.45$, tissues being considered as quasi-incompressible. A higher stiffness 
equal to $E=10 \mathrm{kPa}$ is used for the tumor, close to the value chosen by Miller and Lu (2013). So far, these biomechanical parameters are neither patient-specific nor dependent on the type and location of the tumor.

\subsubsection{Gravity-induced pre-stress computation}

The brain shape segmented from preoperative MRI corresponds to an equilibrium state between internal and external forces. If external forces are applied without accounting for the internal ones, an other equilibrium state will be reached, not corresponding any more to the segmented shape. Since internal forces are unknown, the external ones (e.g. gravity) are then ignored by most of the biomechanical models. However, gravity is one of the main causes of the craniotomy-induced brain-shift.

An algorithm to compute the gravity-induced internal pre-stress is then proposed by Morin et al. (2015) for a highly deformable model of the brain. Rest positions are iteratively computed so that, when the gravity is applied, the reached equilibrium state geometrically corresponds to the segmented shape. Impact of this internal pre-stress when free loads (forces) are applied is studied, pointing out that a pre-stressed model is far more stiff than a stress-free one. As a consequence, the amplitude of node displacements is significantly reduced, while the stress is higher.

In our specific context of vessel-based deformations, loads are imposed through displacements. Therefore, the simulated node displacements should be almost insensitive to pre-stressing. Still, a difference could be expected in terms of stress, also this has yet to be studied. In a wider framework however, for example with pressure loads or less constrained deformations, this pre-stressing step should really by carried out to account for gravity.

\section{Results}

The vessel-based brain-shift compensation method detailed above has been tested on one clinical case of low grade tumor located in the left frontal lobe. Data were collected by the Norwegian National Advisory Unit for Ultrasound and Image-Guided Therapy (www.usigt.org) with Sonowand Invite (Sonowand AS, Trondheim, Norway). The biomechanical FE brain model, composed of 3316 nodes and 16318 tetrahedral elements, was developed using the simulation framework Sofa (www.sofa-framework.org).

Quantitative results were presented by Morin et al. (2016), showing the ability of the method to compensate for brain-shift while being compatible with a surgical process in terms of execution times. Our method was also compared to the modified ICP algorithm proposed by Reinertsen et al. (2014). The brain-shift was measured on blood vessels using landmarks identified on the preoperative MRA and intraoperative US images. Its average value was then reduced from $3.98 \mathrm{~mm}$ to 1.38 


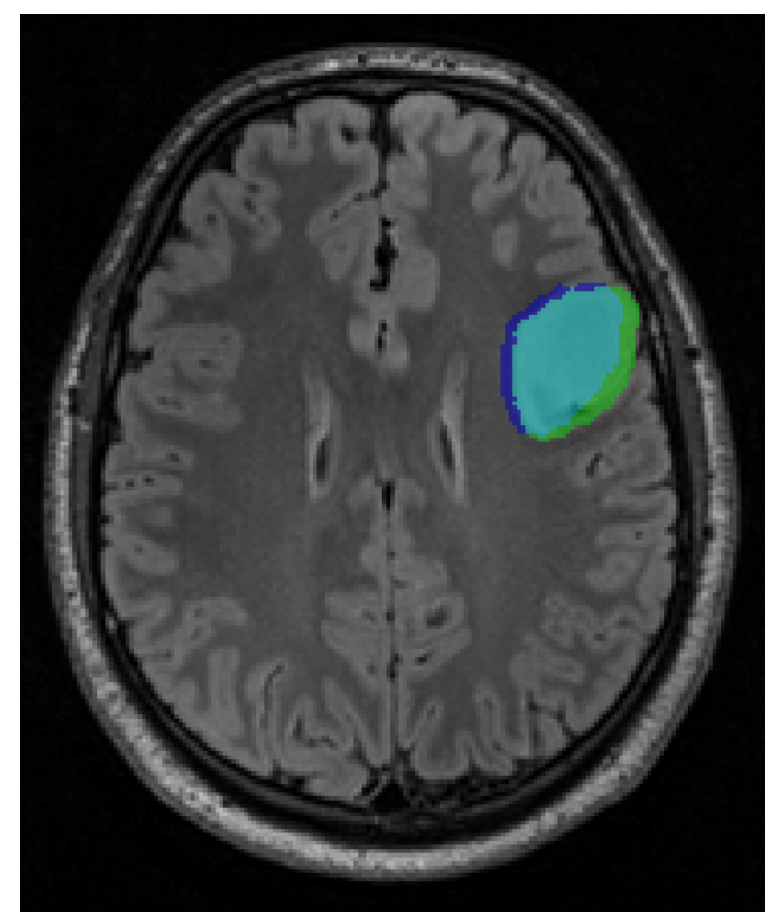

(a) Axial view

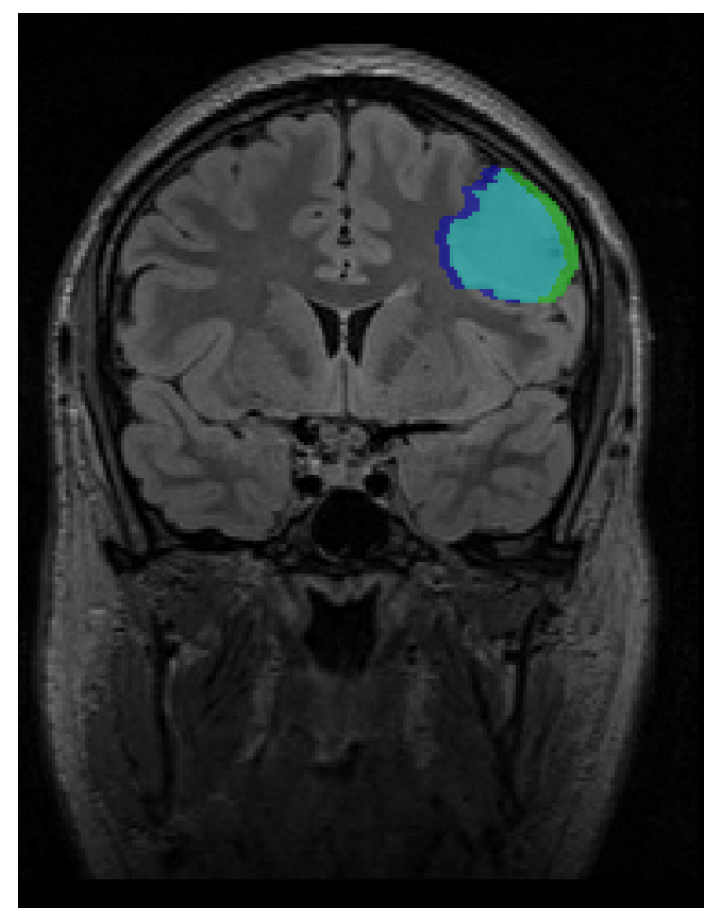

(b) Coronal view

Figure 7.4: Virtual rendering of the brain-shift on the pre-operative MRI, including the segmented tumor (green), the segmented tumor deformed with our method (dark blue) and their intersection (light blue)

$\mathrm{mm}$ with our elastic registration method, against $2.59 \mathrm{~mm}$ with the modified ICP. In addition, the tumor was segmented on the preoperative MRI and intraoperative B mode US images by two medical experts from the St Olav Hospital (Trondheim, Norway). The MR segmentation was updated using the two methods and resulting images were compared to the B-mode US segmentation. A better overlap using our elastic registration was shown quantitatively and qualitatively.

Finally, preoperative and updated tumor segmentation are displayed on axial and coronal views of the MRI acquired prior to surgery (Figure 7.4). An important gap can be seen, pointing out the necessity to provide some solutions to accurately estimate the tumor displacement and deformation. So far, only the craniotomy-induced brain-shift at the beginning of the surgery is accounted for. Future works thus include correcting the brain-shift later into the procedure, as well as taking into account the deformation due to the tumor resection.

\section{Conclusion}

As shown in this survey, brain soft tissues behavior is extremely complex with non-linear, inhomogeneous and subject-specific biomechanical properties. In addition, brain modeling and simulation have many applications in medicine, with a large range of constraints and specificity: objectives, available data, boundary conditions, user interactions, requirements in terms of execution-time or 
clinical practicability, etc. Many models have been proposed in the literature, sometimes with inconsistent or contradictory laws and parameters. The modeling of this organ therefore remains challenging and should be specifically designed according to the targeted medical application.

Acknowledgments. We would like to thank Ingerid Reinertsen and the SINTEF Medical Technology (Trondheim, Norway) for collaboration and providing us with the medical images.

This study was partially funded by the French ANR within the references ANR-11-LABX0004 (Labex CAMI) and ANR-11-INBS-0006 (Infrastructure d'avenir en Biologie Santé) and by a France-Norway partnership (PHC Aurora 2015/Research Council of Norway).

\section{References}

Bayly PV, Clayton EH, Genin GM. Quantitative Imaging Methods for the Development and Validation of Brain Biomechanics Models. Annual review of biomedical engineering 2012;14:36996.

Bilger A, Dequidt J, Duriez C, Cotin S. Biomechanical simulation of electrode migration for deep brain stimulation. Medical Image Computing and Computer-Assisted Intervention - MICCAI 2011 2011;14(1):339-46.

Bilston LE. Brain tissue mechanical properties. In: Biomechanics of the brain. Springer; 2011. p. $69-89$.

Bucki M, Palombi O, Bailet M, Payan Y. Doppler Ultrasound Driven Biomechanical Model of the Brain for Intraoperative Brain-Shift Compensation: A Proof of Concept in Clinical Conditions. In: Soft Tissue Biomechanical Modeling for Computer Assisted Surgery. Springer; 2012. p. 13565.

Budday S, Nay R, de Rooij R, Steinmann P, Wyrobek T, Ovaert TC, Kuhl E. Mechanical properties of gray and white matter brain tissue by indentation. Journal of the mechanical behavior of biomedical materials 2015;46:318-30.

Budday S, Raybaud C, Kuhl E. A mechanical model predicts morphological abnormalities in the developing human brain. Scientific reports 2014;4.

Castellano-Smith AD, Crum WR, Hill DLG, Thacker NA, Bromiley PA. Biomechanical simulation of atrophy in MR images. Medical Imaging 2003;:481-90.

Chatelin S, Constantinesco A, Willinger R. Fifty years of brain tissue mechanical testing: From in vitro to in vivo investigations. Biorheology 2010;47(5-6):255-76. 
Chatelin S, Vappou J, Roth S, Raul JS, Willinger R. Towards child versus adult brain mechanical properties. Journal of the mechanical behavior of biomedical materials 2012;6:166-73.

Chen I, Coffey AM, Ding S, Dumpuri P, Dawant BM, Thompson RC, Miga MI. Intraoperative Brain Shift Compensation: Accounting for Dural Septa. IEEE Transcations on Biomedical Engineering 2011;58(3):499 - 508 .

Clatz O, Delingette H, Bardinet E, Dormont D, Ayache N. Patient-specific biomechanical model of the brain: application to Parkinson's disease procedure. Surgery Simulation and Soft Tissue Modeling 2003;:321-31.

Clatz O, Delingette H, Talos IF, Golby AJ, Kikinis R, Jolesz FA, Ayache N, Warfield SK. Robust Nonrigid Registration to Capture Brain Shift From Intraoperative MRI. IEEE Transactions on Medical Imaging 2005;24(11):1417-27.

Courtecuisse H, Peterlik I, Trivisonne R, Duriez C, Cotin S. Constraint-based simulation for nonrigid real-time registration. Medicine Meets Virtual Reality 21: NextMed/MMVR21 2014;196:7682.

De Lorenzo C, Papademetris X, Staib LH, Vives KP, Spencer DD, Duncan JS. Volumetric Intraoperative Brain Deformation Compensation: Model Development and Phantom Validation. IEEE Transactions on Medical Imaging 2012;31(8):1607-19.

Dequidt J, Coevoet E, Thines L, Duriez C. Vascular neurosurgery simulation with bimanual haptic feedback. In: 12th Workshop on Virtual Reality Interaction and Physical Simulation. 2015. .

Dorsey ER, Constantinescu R, Thompson JP, Biglan KM, Holloway RG, Kieburtz K, Marshall FJ, Ravina BM, Schifitto G, Siderowf A, Tanner CM. Projected number of people with Parkinson disease in the most populous nations, 2005 through 2030. Neurology 2007;68(5):384-6.

Dumpuri P, Thompson RC, Sinha TK, Miga MI. Automated Brain Shift Correction Using A Pre-computed Deformation Atlas. Medical Imaging 2006;:61411F-.

Echegaray G, Herrera I, Aguinaga I, Buchart C, Borro D. A Brain Surgery Simulator. Computer Graphics and Applications, IEEE 2014;34(3):12-8.

Fuerst B, Wein W, Müller M, Navab N. Automatic ultrasound-MRI registration for neurosurgery using the 2d and 3d LC2 Metric. Medical Image Analysis 2014;(18):1312-9.

Gerard IJ, Kersten-Oertel M, Petrecca K, Sirhan D, Hall JA, Collins DL. Brain shift in neuronavigation of brain tumors: A review. Medical Image Analysis 2017;35:403-20. 
Goriely A, Geers MGD, Holzapfel GA, Jayamohan J, Jérusalem A, Sivaloganathan S, Squier W, van Dommelen JAW, Waters S, Kuhl E. Mechanics of the brain: perspectives, challenges, and opportunities. Biomechanics and modeling in mechanobiology 2015;14(5):931-65.

Green MA, Sinkus R, Bilston LE. High Resolution 3d Brain MR-Elastography. Proc Intl Soc Mag Reson Med 2006;14.

Hamhaber U, Sack I, Papazoglou S, Rump J, Klatt D, Braun J. Three-dimensional analysis of shear wave propagation observed by in vivo magnetic resonance elastography of the brain. Acta Biomaterialia 2007;3(1):127-37.

Hastreiter P, Rezk-Salama C, Soza G, Bauer M, Greiner G, Fahlbush R, Ganslandt O, Nimsky C. Strategies for brain-shift evaluation. Medical Image Analysis 2004;8:447-64.

Hrapko M, van Dommelen JAW, Peters GWM, Wismans JSHM. The Influence of Test Conditions on Characterization of the Mechanical Properties of Brain Tissue. Journal of Biomechanical Engineering 2008;130(3):031003.

Hu J, Jin X, Lee JB, Zhang L, Chaudary V, Guthikonda M, Yang KH, King AI. Intraoperative brain shift prediction using a $3 \mathrm{~d}$ inhomogeneous patient-specific finite element model. Journal of neurosurgery 2007;106(1):164-9.

Kaster T, Sack I, Samani A. Measurement of the hyperelastic properties of ex vivo brain tissue slices. Journal of Biomechanics 2011;44(6):1158-63.

Kruse SA, Dresner MA, Rossman PJ, Felmlee JP, Jack CR, Ehman RL. Palpation of the Brain Using Magnetic Resonance Elastography. International Society for Magnetic Resonance in Medicine $1999 ;: 258$.

Kruse SA, Rose GH, Glaser KJ, Manduca A, Felmlee JP, Jack Jr. CR, Ehman RL. Magnetic resonance elastography of the brain. NeuroImage 2008;39(1):231-7.

Kyriacou SK, Davatzikos C, Zinreich SJ, Bryan RN. Nonlinear Elastic Registration of Brain Images with Tumor Pathology Using a Biomechanical Model. IEEE Transactions on Medical Imaging $1999 ; 18(7): 580-92$.

Laksari K, Shafieian M, Darvish K. Constitutive model for brain tissue under finite compression. Journal of Biomechanics 2012;45(4):642-6.

McCracken PJ, Manduca A, Felmlee JP, Ehman RL. Mechanical Transient-Based Magnetic Resonance Elastography. Magnetic Resonance in Medicine 2005;53:628-39. 
Mercier L, Fonov V, Haegelen C, Del Maestro RF, Petrecca K, Collins DL. Comparing two approaches to rigid registration of three-dimensional ultrasound and magnetic resonance images for neurosurgery. International journal of computer assisted radiology and surgery 2012;7(1):125-36.

Miga MI, Paulsen KD, Hoopes J, Kennedy FE, Hartov A, Roberts DW. In Vivo Quantification of a Homogeneous Brain Deformation Model for Updating Preoperative Images During Surgery. IEEE Transactions on Biomedical Engineering 2000;47(2):266-73.

Miga MI, Sun K, Chen I, Clements LW, Pheiffer TS, Simpson AL, Thompson RC. Clinical evaluation of a model-updated image-guidance approach to brain shift compensation: experience in 16 cases. International journal of computer assisted radiology and surgery 2015;11(8):1467-74.

Miller K, Chinzei K. Mechanical properties of brain tissue in tension. Journal of Biomechanics 2002;35:483-90.

Miller K, Joldes GR, Lance D, Wittek A. Total Lagrangian explicit dynamics finite element algorithm for computing soft tissue deformation. COMMUNICATIONS IN NUMERICAL METHODS IN ENGINEERING 2007;23:121-34.

Miller K, Lu J. On the prospect of patient-specific biomechanics without patient-specific properties of tissues. Journal of the mechanical behavior of biomedical materials 2013;27:154-66.

Miller K, Wittek A, Joldes GR. Biomechanics of the brain for computer-integrated surgery. Acta of Bioengineering and Biomechanics 2002;12(2):25-37.

Mohammadi A, Ahmadian A, Darbandi Azar A, Darban Sheykh A, Amiri F, Alirezaie J. Estimation of intraoperative brain shift by combination of stereovision and doppler ultrasound: phantom and animal model study. International journal of computer assisted radiology and surgery $2015 ; 10(11): 1753-64$.

Morin F, Courtecuisse H, Chabanas M, Payan Y. Rest shape computation for highly deformable model of brain. Computer Methods in Biomechanics and Biomedical Engineering 2015;18(Sup1):2006-7.

Morin F, Reinertsen I, Courtecuisse H, Palombi O, Munkvold B, Bø HK, Payan Y, Chabanas M. Vessel-based brain-shift compensation using elastic registration driven by a patient-specific finite element model. In: International Conference on Image Processing and Computer-Assisted Intervention - IPCAI 2016. 2016. . 
Muthupillai R, Lomas DJ, Rossman PJ, Greenleaf JF, Manduca A, Ehman RL. Magnetic resonance elastography by direct visualization of propagating acoustic strain waves. Science 1995;269(5232):1854-7.

Müller M, Dorsey J, McMillan L, Jagnow R, Cutler B. Stable real-time deformations. Proceedings of ACM SIGGRAPH Symposium on Computer Animation (SCA) 2002;:49-54.

Ommaya AK. Mechanical Properties of Tissues of the Nervous System. Journal of Biomechanics 1968;1(2):127-38.

Ostrom QT, Gittleman H, Fulop J, Liu M, Blanda R, Kromer C, Wolinsky Y, Kruchko C, BarnholtzSloan JS. CBTRUS Statistical Report: Primary Brain and Central Nervous System Tumors Diagnosed in the United States in 2008-2012. Neuro-Onco 2015;17:1-62.

Paulsen KD, Miga MI, Kennedy FE, Hoopes J, Hartov A, Roberts DW. A Computational Model for Tracking Subsurface Tissue Deformation During Stereotactic Neurosurgery. IEEE Transactions on Biomedical Engineering 1999;46(2):213-25.

Prange MT, Margulies SS. Regional, Directional, and Age-Dependent Properties of the Brain Undergoing Large Deformation. Journal of biomechanical engineering 2002;124(2):244-52.

Prastawa M, Bullit E, Gerig G. Simulation of brain tumors in MR images for evaluation of segmentation efficacy. Medical Image Analysis 2009;13(2):297-311.

Pudenz RH, Shelden CH. The Lucite Calvarium-A Method for Direct Observation of the Brain: II. Cranial Trauma and Brain Movement. Journal of neurosurgery 1946;3(6):487-505.

Reinertsen I, Lindseth F, Askeland C, Iversen DH, Unsgard G. Intra-operative correction of brainshift. Acta Neurochirurgica 2014;156:1301-10.

Rivaz H, Collins DL. Deformable registration of preoperative MR, pre-resection ultrasound, and post-resection ultrasound images of neurosurgery. International journal of computer assisted radiology and surgery 2015;10(7):1017-28.

Rutland-Brown W, Langlois J, Thomas K, Xi Y. Incidence of traumatic brain injury in the United States. 2003. Journal of Head Tram Rehabilitation 2006;21(6):544-8.

Sack I, Beierbach B, Wuerfel J, Dieter K, Hamhaber U, Papazoglou S, Martus P, Braun J. The impact of aging and gender on brain viscoelasticity. NeuroImage 2009;46(3):652-7.

Sase K, Fukuhara A, Tsujita T, Konno A. GPU-accelerated surgery simulation for opening a brain fissure. Robomech Journal 2015;2(1):1-16. 
Schiavone P, Chassat F, Boudou T, Promayon E, Valdivia F, Payan Y. In vivo measurement of human brain elasticity using a light aspiration device. Medical Image Analysis 2009;13:673-8.

Soza G, Grosso R, Nimsky C, Greiner G, Hastreiter P. Estimating Mechanical Brain Tissue Properties with Simulation and Registration. Medical Image Computing and Computer-Assisted Intervention-MICCAI 2004 2004;:276-83.

Sun K, Pheiffer TS, Simpson AL, Weis JA, Thompson RC, Miga MI. Near Real-Time Computer Assisted Surgery for Brain Shift Correction Using Biomechanical Models. IEEE Journal of Translational Engineering in Health and Medicine 2014;2:1-13.

Takizawa H, Sugiura K, Baba M, Miller JD. Analysis of Intracerebral Hematoma Shapes by Numerical Computer Simulation Using the Finite Element Method. Neurologia medico-chirurgica $1994 ; 34(2): 65-9$.

Uffmann K, Maderwald S, de Greiff A, Ladd ME. Determination of Gray and White Matter Elasticity with MR Elastography. Proc Intl Soc Mag Reson Med 2004;11.

Valencia A, Benjamin B, Ortega JH. Modeling of Brain Shift Phenomenon for Different Craniotomies and Solid Models. Journal of Applied Mathematics 2012;2012.

Vang Hausen K, Vlhelm Larsen O. Using Region-of-Interest Based Finite Element Modelling for Brain-Surgery Simulation. Medical Image Computing and Computer-Assisted Interventation MICCAI'98 1998;:305-16.

Vigneron LM, Noels L, Warfield SK, Verly JG, Robe PA. Serial FEM/XFEM-Based Update of Preoperative Brain Images Using Intraoperative MRI. International Journal of Biomedical Imaging 2012;2012:872783.

Wittek A, Hawkins T, Miller K. On the unimportance of constitutive models in computing brain deformation for image-guided surgery. Biomechanics and modeling in mechanobiology 2009;8(1):7784.

Wittek A, Miller K, Kikinis R, Warfield SK. Patient-specific model of brain deformation: Application to medical image registration. Journal of Biomechanics 2007;40(4):919-29.

Xu L, Lin Y, Xi Z, Shen H, Gao P. Magnetic Resonance Elastography of Brain Tumors: Preliminary Results. Acta Radiologica 2007;48(3):327-30.

Yousefi H, Ahmadian A, Khodadad D, Saberi H, Daneshmehr A. An optimised linear mechanical model for estimating brain shift caused by meningioma tumours. International Journal of Biomedical Science and Engineering 2013;1(1):1-9. 